\title{
Ciclos Clássicos num Ambiente de Racionalidade Limitada
}

Jaylson Jair da Silveira

\author{
Professor do Departamento de Economia/Faculdade \\ de Ciências e Letras/Campus de Araraquara/ \\ Universidade Estadual Paulista (UNESP)
}

\section{RESUMO}

O presente trabalho toma como ponto de partida o modelo macrodinâmico de Richard M. Goodwin, apresentado no clássico artigo "A Growth Cycle" (1967). Neste modelo a interação entre distribuição de renda e acumulação de capital gera um ciclo de crescimento que oscila em torno de uma trajetória de crescimento equilibrado. $O$ objetivo do presente trabaIho é investigar a possibilidade de existência de ciclos goodwinianos com heterogeneidade de salários e de taxas de lucro. Essa análise é realizada considerando o processo de concorrência entre trabalhadores num ambiente de racionalidade limitada. Para formalizar o processo de concorrência entre trabalhadores foram utilizados dois recursos: (i) a "alegoria das ilhas", que tem sido amplamente adotada na macroeconomia moderna, a fim de apreender situações de informação imperfeita; e (ii) os instrumentos analíticos desenvolvidos pela teoria dos jogos evolucionários aplicada a ambientes sociais. O modelo construído combina o modelo de Goodwin com uma formalização estilizada do fluxo de trabalho como um processo de imitação num ambiente de racionalidade limitada (dinâmica de replicação num contexto social). Neste modelo demonstra-se a possibilidade de o sistema econômico não convergir para um estado de equilíbrio de longo prazo e a possibilidade do sistema econômico convergir para um ciclo goodwiniano caracterizado por heterogeneidade de salários e de taxas de lucro.

\section{PALAVRAS-CHAVE}

ciclos clássicos, jogo evolucionário, racionalidade limitada

ABSTRACT

This paper starts from the macrodynamical model developed by Richard M. Goodwin, which was first shown in the seminal paper "A Growth Cycle" (Goodwin, 1967). In this model it was described an interaction between income-distribution and capital accumulation that generates a growth cycle swinging along a balanced growth path. The objective of this work is to investigate the possibility of existence of goodwinian cycles with heterogeneity in wage and in profit rates. This task is carried out considering the competitive process among workers within a context of bounded-rationality. The competitive process was modelled on the bases of two tools: (i) "islands's allegory" which has been broadly adopted in the modern macroeconomics in order to apprehend situations of imperfect information; and (ii) the analytic instruments developed by the evolutionary game theory applied to the social environment. The model built combines Goodwin's model with a stylized formalization of the workers flow as a process of imitation within a context of bounded-rationality

(social-based replicator dynamics). In this model two results are demonstrated: the possibility of the economy not converge to a long run equilibrium and the possibility of the economy to present $a$ goodwinian cycle with heterogeneity in wage and in profit rates.

KEY WORDS growth cycle, evolutionary game, bounded rationality

\author{
JEL Classification
}




\section{INTRODUÇÃO}

Goodwin (1967), no artigo seminal "A growth cycle", apresenta um modelo macrodinâmico de inspiração clássica/marxista no qual a interação entre distribuição de renda e acumulação de capital faz emergir um ciclo de crescimento em torno de uma trajetória de crescimento equilibrado. O caráter inovador deste modelo reside na combinação de uma estrutura típica de um modelo de crescimento clássico com uma curva de Phillips real, que tenta captar a idéia de que os salários pressionam fortemente as taxas de lucro nos períodos de intensa acumulação de capital. O modelo de Goodwin gerou um programa de pesquisa que busca incorporar à estrutura geradora de ciclos goodwiniana questões como inflação, progresso técnico, hipótese de salário-eficiência etc. Versões desagregadas do modelo de Goodwin foram propostas (vide GOODWIN et al., 1984, p. 67-72 e SATO, 1985), sendo nelas utilizada a hipótese simplificadora de que o processo de concorrência entre capitais e entre trabalhadores mantinha as taxas de lucro e os salários equalizados durante o desenrolar do ciclo. ${ }^{1}$

No presente trabalho avalia-se a possibilidade de existência de um ciclo goodwiniano com heterogeneidade de salários e de taxas de lucro quando se leva em consideração, além da barganha entre trabalhadores e firmas por uma maior participação na renda, o processo migratório de trabalhadores desencadeado por diferenciais de salário. Partindo do princípio de que os trabalhadores se preocupam com a estrutura de salários relativos e que informações sobre esta são imperfeitas, o fluxo migratório de trabalhadores entre mercados de trabalho é formalizado, utilizando instrumentos analíticos da teoria dos jogos evolucionários, ${ }^{2}$ como uma dinâmica de seleção fundamentada num processo de imitação.

1 Para mais detalhes sobre o modelo de Goodwin e o programa de pesquisa a ele associado, consulte SILVEIRA (2001, cap. 1).

2 Sobre tal teoria consulte HOFBAUER \& SIGMUND (1998), SAMUELSON (1997), VEGAREDONDO (1996) ou WEIBULL (1995). 
Este texto está organizado como segue: na seção a seguir estabelece-se a estrutura do modelo; na segunda seção efetua-se uma análise de estabilidade local, baseada na técnica de linearização, em torno dos estados de equilíbrio de longo prazo; na terceira seção demonstra-se, com o auxílio do teorema de bifurcação de Poincaré-Andronov-Hopf, a possibilidade de existência de um ciclo com heterogeneidade de salários e de taxas de lucro; na última seção fecha-se o artigo.

\section{ESTRUTURA DO MODELO}

Como é habitual na modelagem macroeconômica, pode-se construir uma alegoria como cenário básico onde possa ser estudada a interação entre acumulação de capital, distribuição de renda e o fluxo intersetorial de trabalhadores desencadeado pela heterogeneidade de salários. Considere um sistema econômico formado por duas ilhas habitadas pelo mesmo tipo de trabalhadores, ${ }^{3}$ contratados por firmas que produzem um bem homogêneo sujeitas à mesma tecnologia. $\mathrm{O}$ bem produzido na ilha $\mathrm{l}$ é igual ao produzido na ilha 2. Nos intervalos entre períodos de produção ${ }^{4}$ consecutivos, os trabalhadores realizam comparações de salários em pares compostos aleatoriamente. Efetuadas as comparações, cada trabalhador escolhe uma das duas opções: permanecer na ilha na qual se encontrava (empregado ou desempregado) no período de produção anterior ou realizar uma migração para a outra ilha. Exclui-se, por hipótese, a possibilidade de que um trabalhador possa ofertar sua força de trabalho nas duas ilhas simultaneamente. Após todos os trabalhadores decidirem em que ilha irão oferecer suas forças de trabalho, as populações das ilhas estarão determinadas. Os trabalhadores negociam coletivamente com as firmas o salário que será pago no período de produção que está para ser iniciado. Acordado o nível de salário em cada ilha, as firmas determinam a quantidade empregada de trabalho. No

3 No sentido de que, do ponto de vista das firmas, um trabalhador da ilha 1 possui as mesmas características de um trabalhador da ilha 2.

4 Iguais e sincronizados entre as ilhas. 
próximo intervalo entre períodos de produção consecutivos essa seqüência de eventos recomeça.

\subsection{Fluxo Migratório de Trabalhadores como uma Dinâmica de Seleção}

Considerando o cenário descrito anteriormente, coloca-se a seguinte questão: dadas as taxas setoriais de emprego e a estrutura de salários reais relativos, como se pode estimar a variação da distribuição de trabalhadores entre as ilhas entre dois períodos de produção sucessivos?

Assumir-se-á que todos os trabalhadores estão dispostos a trabalhar uma dada jornada de trabalho por qualquer salário real igual ou superior a um valor ditado pelo custo de subsistência (normalizado em zero). Além disso, admitir-se-á, à la Keynes, que os trabalhadores estão preocupados com seus salários relativos. É suposto que a comparação de salários será feita dois a dois a partir de indivíduos selecionados aleatoriamente de uma população suficientemente grande.

Sejam $N$ o número total de trabalhadores do sistema econômico e $N_{i}$ o número de trabalhadores na ilha $i, \operatorname{com} i=1,2$. Num dado momento, existem $N_{1}$ trabalhadores na ilha 1 . Uma fração $\nu_{1}$ do total de trabalhadores na ilha 1 está empregada e, portanto, $1-v_{1}$ está desempregada. ${ }^{5}$ Logo, há na ilha 1 $N_{1} v_{1}$ trabalhadores empregados e $N_{1}\left(1-v_{1}\right)$ desempregados. Analogamente, existem $N_{2}$ trabalhadores na ilha 2. Uma fração $v_{2}$ destes trabalhadores encontra-se empregada, ao passo que a fração restante $1-v_{2}$ encontra-se desempregada. Assim, $\mathrm{N}_{2} v_{2}$ trabalhadores da ilha 2 estão empregados e $N_{2}\left(1-v_{2}\right)$ desempregados.

É conveniente normalizar $N$ como sendo igual a l. Dessa maneira, $n_{i}=N_{i} / N$ passa a designar o número de trabalhadores na ilha $i$ em unidades de população. Desde de que os trabalhadores são, por hipótese,

$5 \mathcal{V}_{i}$ é a taxa de emprego da $i$-ésima ilha, ou seja, a razão entre as respectivas quantidades empregada $\left(L_{i}\right)$ e ofertada $\left(N_{i}\right)$ de trabalho nesta ilha. Está se supondo implicitamente que todos os trabalhadores de uma ilha têm iguais chances de serem contratados. Cabe lembrar que do ponto de vista dos trabalhadores $v_{i}=1$ se $L_{i}>N_{i}$ para $i=1,2$. 
homogêneos, suas chances de serem contratados são iguais. Dessa forma, a probabilidade de um trabalhador encontrar-se empregado na ilha 1 , desempregado na ilha 1 , empregado na ilha 2 ou desempregado na ilha 2 é, respectivamente, $n_{1} v_{1}, n_{1}\left(1-v_{1}\right),\left(1-n_{1}\right) v_{2}$ e $\left(1-n_{1}\right\rangle\left(1-v_{2}\right)$.

Como $n_{1}+n_{2}=1$, basta considerar a evolução de uma dessas variáveis. Tomar-se-á como referência a ilha 1 . A taxa de variação estimada da proporção da população de trabalhadores ofertando sua força de trabalho na ilha 1 , num intervalo infinitesimal de tempo, será a diferença entre o total estimado de trabalhadores imigrantes para a ilha 1 (influxo) e o total estimado de trabalhadores emigrantes para a ilha 2 (efluxo), ou seja: ${ }^{6}$

$$
\dot{n}_{1}=\text { influxo de trabalhadores - efluxo de trabalhadores. }
$$

É importante observar que, se um trabalhador na ilha $i$ compara seu salário com um outro trabalhador na ilha $i$, ele não tem informação sobre o mercado de trabalho $j \neq i$ e, portanto, não tem referência para avaliar o desempenho relativo de sua atual estratégia. Neste caso, supõe-se que o trabalhador não altera a sua estratégia. Assim, considera-se que a comparação de salários entre trabalhadores que atuam em ilhas distintas é condição necessária, embora não suficiente, para que ocorra uma migração.

$\mathrm{Na}$ Figura 1 encontram-se árvores de possibilidades que sintetizam os encontros possíveis do ponto de vista dos trabalhadores da ilha l(Figura l-a) e da ilha 2 (Figura l-b). Considere o evento $E_{1}$, o qual representa a seguinte ocorrência: um trabalhador empregado na ilha $i$ compara seu salário com o de um trabalhador empregado na ilha $j$, com $j \neq i$. Como, num dado instante, há, em unidades de população, $n_{i} v_{i}$ trabalhadores empregados na ilha $i$ e a probabilidade de encontrar um trabalhador empregado na ilha $j$ é $n_{j} v_{j}$, então o número estimado (em unidades de população) de trabalhadores empregados na ilha $i$ que irão comparar seus salários com

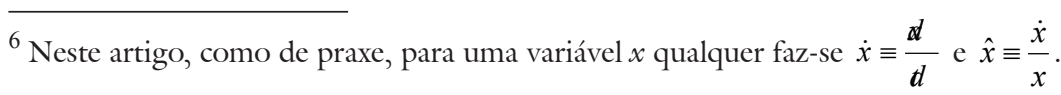


os salários dos trabalhadores empregados na ilha $j$ é $n_{i} v_{i} n_{j} v_{j}$. Os demais eventos representados nas árvores de possibilidades da Figura 1 podem ser interpretados de maneira similar.

\section{FIGURA 1}

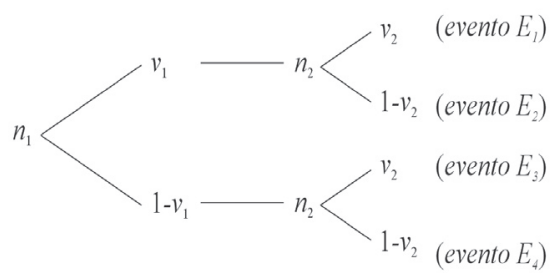

(a)

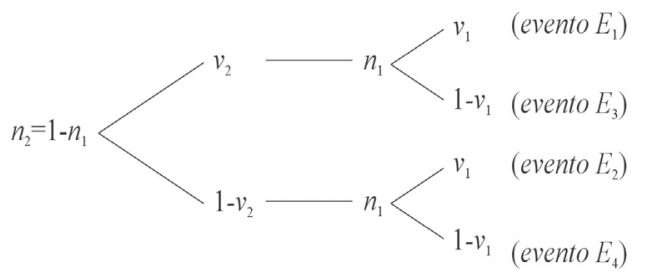

(b)

Seja $\omega_{\mathrm{i}}$ o salário real na $i$-ésima ilha. Na comparação de rendimentos entre trabalhadores ligados a ilhas distintas podem surgir, a priori, quatro diferenciais, conforme sintetizado na Tabela 1.

TABELA 1 - DIFERENCIAIS DE RENDIMENTO

\begin{tabular}{llcllll}
\hline \multirow{2}{*}{ Caso } & \multicolumn{2}{c}{ Trabalhador na llha 1 } & & \multicolumn{2}{c}{ Trabalhador na ilha 2 } & \multirow{2}{*}{$R_{1}-R_{2}$} \\
\cline { 2 - 3 } & Situação & Rendimento $\left(R_{1}\right)$ & & Situação & Rendimento $\left(R_{2}\right)$ & \\
\hline $\mathrm{C}_{1}$ & Empregado & $\omega_{1}$ & Empregado & $\omega_{2}$ & $\omega_{1}-\omega_{2}$ \\
$\mathrm{C}_{2}$ & Empregado & $\omega_{1}$ & Desempregado & 0 & $\omega_{1}$ \\
$\mathrm{C}_{3}$ & Desempregado & 0 & Empregado & $\omega_{2}$ & $-\omega_{2}$ \\
$\mathrm{C}_{4}$ & Desempregado & 0 & Desempregado & 0 & 0 \\
\hline
\end{tabular}

A comparação por parte de um trabalhador da ilha $i$ de seu rendimento com o rendimento de um trabalhador da ilha $j(j \neq i)$ é apenas uma condição necessária para a ocorrência da mudança de estratégia por parte do primeiro. Uma outra condição necessária para a mudança de estratégia é que $R_{j}>R_{i}$. Quando um trabalhador da ilha $i$ compara seu rendimento com o rendimento de um trabalhador da ilha $j$, com $i \neq j$, e $R_{j}>R_{i}$, a efetivação da mudança de estratégia dependerá da magnitude do diferencial 
de rendimento. Quanto maior este diferencial, maior é o incentivo para o trabalhador da ilha $i$ buscar um novo emprego na ilha $j$. Mais precisamente, para $i \neq j$ e $R_{j}>R_{i}$, a proporção de trabalhadores na ilha $i$ que efetivamente imigrará para a ilha $j$ será suposta diretamente proporcional ao diferencial ${ }^{7}$ $R_{j}-R_{i}$.

\section{Influxo de Trabalhadores na Ilha 1}

Com base na Figura l-b e na Tabela l pode-se determinar uma estimativa do influxo de trabalhadores na ilha 1 . Relacionado ao evento $E_{1}$ da Figura l-b tem-se o caso $C_{1}$ da Tabela 1. Se $R_{1}-R_{2}=\omega_{1}-\omega_{2}>0$, o influxo estimado para a ilha 1 de trabalhadores empregados na ilha 2 , que compararam o seu salário com o salário de um trabalhador empregado na ilha 1, é, então, diretamente proporcional ao diferencial salarial, mais precisamente, $\left(1-n_{1}\right) v_{2} n_{1} v_{1}\left(\omega_{1}-\omega_{2}\right)$. Se $R_{1}-R_{2}=\omega_{1}-\omega_{2} \leq 0$, a quantidade de trabalhadores imigrantes para a ilha 1 será nula. De forma mais compacta, a expressão a seguir apreende as duas possibilidades:

$$
\left(1-n_{1}\right) v_{2} n_{1} v_{1} \max \left\{\omega_{1}-\omega_{2}, 0\right\} .
$$

Ao evento $E_{2}$ da Figura 1-b está relacionado o caso $C_{2}$ da Tabela 1. Quando os trabalhadores desempregados na ilha 2 comparam seus rendimentos com os dos trabalhadores empregados na ilha 1 , o diferencial de rendimentos é igual a $\omega_{1}$. Portanto, a proporção de trabalhadores que efetivamente imigrará é diretamente proporcional a $\omega_{1}$ :

$$
\left(1-n_{1} \chi 1-v_{2}\right) n_{1} v_{1} \omega_{1} \text {. }
$$

7 Poder-se-ia expressar este diferencial de rendimentos em unidades do máximo diferencial de salários entre os diferenciais salariais médios possíveis a priori, tal que $\left|R_{j}-R_{i}\right| \in[0,1] \in \mathfrak{R}$. Isto não alteraria os resultados obtidos nesta seção. 
O evento $E_{3}$ da Figura 1-b está relacionado ao caso $C_{3}$ da Tabela 1. Nesse caso a condição necessária para a imigração, $R_{1}-R_{2}>0$, não é satisfeita, conseqüentemente, não haverá imigração para a ilha 1 .

Finalmente, quando ocorre o evento $E_{4}$ da Figura 1-b, ligado ao caso $C_{4}$ (desempregado comparando com desempregado) da Tabela 1 , não se efetivará qualquer imigração de trabalhadores da ilha 2 para a ilha 1 , pois $o$ diferencial de rendimentos é nulo.

Em suma, agregando (2) e (3), o influxo estimado de trabalhadores na ilha 1 é:

$$
\left(1-n_{1}\right) n_{1}\left[v_{2} v_{1} \max \left\{\omega_{1}-\omega_{2}, 0\right\}+\left(1-v_{2}\right) v_{1} \omega_{1}\right]
$$

\section{Efluxo de Trabalhadores na Ilha 1}

De maneira análoga, pode-se utilizar a Figura l-a e a Tabela l para estimar quanto dos $n_{1}$ trabalhadores sairão da ilha $1 \mathrm{em}$ cada intervalo entre períodos de produção consecutivos.

O evento $E_{1}$ da Figura l-a está relacionado ao caso $C_{1}$ da Tabela 1. Se $\omega_{1}-\omega_{2}<0$, o efluxo estimado de trabalhadores empregados na ilha 1 , que comparam o seu salário com o de um trabalhador empregado na ilha 2 , é diretamente proporcional ao diferencial de salários, ou seja, $-n_{1} v_{1}\left(1-n_{1}\right) v_{2}\left(\omega_{1}-\omega_{2}\right)$. Se $\omega_{1}-\omega_{2}>0$, então a quantidade de trabalhadores emigrantes da ilha 1 será nula. Mais sinteticamente:

$$
-n_{1} v_{1}\left(1-n_{1}\right) v_{2} \min \left\{\omega_{1}-\omega_{2}, 0\right\} \text {. }
$$

Ao evento $E_{2}$ da Figura l-a está relacionado o caso $C_{2}$ da Tabela l. Nesta circunstância, a condição necessária para a emigração, $R_{1}-R_{2}<0$, não é encontrada, logo, não se verificará emigração de trabalhadores da ilha 1.

O evento $E_{3}$ da Figura 1-a está associado ao caso $C_{3}$ da Tabela 1. Quando os trabalhadores desempregados na ilha l comparam seus salários com os tra- 
balhadores empregados na ilha 2 , o diferencial de rendimentos é $R_{1}-R_{2}=$ $-\omega_{2}$. Logo, a proporção efetiva de emigrantes neste caso será:

$$
n_{1}\left(1-v_{1} \gamma 1-n_{1}\right) v_{2} \omega_{2}
$$

Como antes, na ocorrência do evento $E_{4}$ da Figura 1-a, ligado ao caso $C_{4}$ da Tabela 1 , não será efetivada qualquer emigração de trabalhadores da ilha 1 , já que o diferencial de rendimentos é nulo.

Resumindo, o efluxo de trabalhadores da ilha l é estimado somando-se (5) e (6):

$$
-n_{1}\left(1-n_{1}\right)\left[v_{1} v_{2} \min \left\{\omega_{1}-\omega_{2}, 0\right\}-\left(1-v_{1}\right) v_{2} \omega_{2}\right]
$$

\section{Dinâmica de Replicação Associada ao Fluxo Migratório de Trabalhadores}

Avaliadas as possibilidades de migração dos trabalhadores, a variação da proporção de trabalhadores ofertando sua força de trabalho na ilha 1 é aproximada pela diferença entre o influxo e o efluxo estimados de trabalhadores nesta ilha, isto é, pela diferença entre (4) e (7):

$\dot{n}_{1}=\left(1-n_{1}\right) n_{1}\left[v_{2} v_{1} \max \left\{\omega_{1}-\omega_{2}, 0\right\}+\left(1-v_{2}\right) v_{1} \omega_{1}\right]+n_{1}\left(1-n_{1}\right)\left[v_{1} v_{2} \min \left\{\omega_{1}-\omega_{2}, 0\right\}-\left(1-v_{1}\right) v_{2} \omega_{2}\right]$.

Como $\max \left\{\omega_{1}-\omega_{2}, 0\right\}+\min \left\{\omega_{1}-\omega_{2}, 0\right\}=\omega_{1}-\omega_{2},(8)$ pode ser simplificada para:

8 A dinâmica de replicação é um tipo específico de dinâmica de seleção (selection dynamics). Esta última é um sistema de equações diferenciais ou em diferenças que apresenta as seguintes características: (i) satisfaz condiçôes mínimas de continuidade de modo que problemas de valor inicial possuam solução única; e (ii) o simplex unitário de dimensão apropriada (seu espaço de estados) é positivamente invariante, o que permite interpretar o estado do sistema como a distribuição de estratégias na(s) população(ões) em análise. Além dessas características fundamentais, uma dinâmica de seleção deve refletir um processo de seleção. Ou seja, para um dado estado da população, se uma estratégia propicia um resultado melhor do que outra, então a sua taxa de crescimento é maior do que a dessa última (payoffmonotonicity, WEIBULL, 1995, p. 144). Há uma outra forma de ligar o estado da população com o diferencial de resultados: qualquer estratégia que apresentar, para um dado estado da população, resultado superior à média, deverá exibir uma taxa de crescimento positiva (payoff positivity, WEIBULL, 1995, p. 149). HOFBAUER \& SIGMUND (1988, p. 47) destacam que equaçôes com a forma da dinâmica de replicação, as quais denominam genericamente de equações de replicação (replicator equations), aparecem em várias áreas da biologia (sociobiologia, evolução macromolecular, ecologia matemática e genéticas de populações). 


$$
\left.\dot{n}_{1}=n_{1}\left(1-n_{1}\right) v_{1} \omega_{1}-v_{2} \omega_{2}\right) .
$$

Essa dinâmica de replicação ${ }^{8}$ espelha formalmente a intuição de que a proporção de trabalhadores que ofertam sua força de trabalho na ilha 1 aumenta, mantém-se constante ou diminui caso o salário médio nesta ilha seja, respectivamente, superior, igual ou inferior ao salário médio na ilha 2.

Uma outra maneira de olhar para a dinâmica de replicação (8-a) é obtida por simples manipulação algébrica, a saber:

$$
\dot{n}_{1}=n_{1}\left\{v_{1} \omega_{1}-\left[n_{1} v_{1} \omega_{1}+\left(1-n_{1}\right) v_{2} \omega_{2}\right]\right\}
$$

A expressão entre colchetes é o salário real médio do sistema econômico como um todo. Portanto, a oferta de trabalho na ilha 1 tende a se expandir se, e somente se, o salário esperado nesta ilha for superior ao salário real médio da economia.

\subsection{Evolução da Quantidade Empregada de Trabalho em cada Ilha}

Nesta subseção adota-se as premissas do modelo agregado de Goodwin (1967) que, aplicadas a cada ilha, determinam as taxas de crescimento das quantidades empregadas de trabalho. Em ambas as ilhas as firmas utilizam como insumos capital e trabalho, os quais são homogêneos e não-específicos, e produzem um único bem homogêneo, que pode ser consumido ou utilizado como bem de capital, sujeito à mesma tecnologia de coeficientes fixos. Ou seja, o sistema produtivo na $i$-ésima ilha produz sujeito à seguinte função de produção:

$$
q_{i}=\min \left\{\frac{1}{b_{k}} k_{i}, \frac{1}{b_{l}} l_{i}\right\}, i=1,2,
$$

na qual $q_{i}$ é a quantidade produzida na $i$-ésima ilha por período de produção, $b_{k}$ a quantidade de capital necessária para produzir uma unidade de produto por período de produção, $k_{i}$ a quantidade utilizada de capital por período de produção na $i$-ésima ilha, $b_{l}$ a quantidade de trabalho necessária para produzir uma unidade de produto por período de produção e $l_{i}$ a 
quantidade empregada de trabalho (normalizada em unidades de população) por período de produção na $i$-ésima ilha.

Supondo que o estoque de capital é plenamente empregado, segue, a partir de (9), que a quantidade empregada de trabalho em cada ilha é determinada pelo nível de produção nesta ilha, $l_{i}=b_{l} q_{i}$, o qual, por sua vez, é determinado pelo respectivo estoque de capital, $q_{i}=k_{i} / b_{k}$. Logo, na $i$-ésima ilha, a quantidade empregada de trabalho é determinada pelo estoque de capital, $l_{i}=\left(b_{l} / b_{k}\right) k_{i}$. Assim, como os coeficientes técnicos são constantes, a taxa de crescimento da quantidade empregada de trabalho na $i$-ésima ilha é igual a:

$$
\hat{l}_{i}=\hat{q}_{i}=\hat{k}_{i}, i=1,2 .
$$

Supondo que os salários são todos gastos em bens de consumo e a massa de lucro na $i$-ésima ilha, $q_{i}-\omega_{1} l_{1}$, é integralmente poupada e automaticamente investida, tem-se que a variação líquida do estoque de capital na $i$-ésima ilha é dada pela massa de lucro:

$$
\dot{k}_{i}=\left(1-\omega_{\mathrm{i}} b_{l}\right) q_{i}, \mathrm{i}=1,2 .
$$

Dividindo ambos os lados de (11) por $k_{i}$ e utilizando (9), obtém-se a taxa de crescimento do estoque de capital na $i$-ésima ilha:

$$
\hat{k}_{i}=\frac{1}{b_{k}}\left(1-\omega_{\mathrm{i}} b_{l}\right), \mathrm{i}=1,2 \text {, }
$$

a qual é igual à taxa de lucro nesta ilha $\left(\rho_{i}\right)$.

Inserindo (12) em (10) tem-se, para um dado nível de salário, as taxas de crescimento das quantidades empregadas de trabalho nas ilhas:

$$
\hat{l}_{i}=\frac{1}{b_{k}}\left(1-\omega_{\mathrm{i}} b_{l}\right), \mathrm{i}=1,2 .
$$




\subsection{Determinação da Estrutura de Salários Relativos}

Goodwin (1967), inspirado no mecanismo do exército industrial de reserva de Marx, postula uma curva de Phillips real na qual a taxa de crescimento do salário real está positivamente relacionada com a taxa de emprego. No presente trabalho esta curva será substituída por uma função barganha, especificada como segue. Supõe-se que o nível do salário real vigente num dado período de produção na $i$-ésima ilha é determinado por um processo de barganha entre as firmas e o sindicato de trabalhadores localizados nesta ilha. O resultado dessa barganha salarial depende da escassez de força de trabalho (medido pela taxa de emprego, $v_{i}$ ) e do tamanho da central sindical (medido pelo número de trabalhadores na ilha, $n_{i}$ ). Esta última variável aparece como argumento da função barganha buscando captar a idéia de que o resultado de uma barganha salarial num determinado setor de um sistema econômico é afetado pela fração de trabalhadores deste sistema que estão negociando de maneira organizada/centralizada neste setor. Ou seja, quanto maior o sindicato de trabalhadores de um determinado setor maior será o impacto de suas ações sobre o sistema econômico como um todo, daí deriva uma fonte adicional de aumento do poder de barganha deste sindicato. Formalmente, à $i$-ésima ilha está associada uma função barganha estritamente quase-côncava:

$$
\omega_{\mathrm{i}}=\phi\left(n_{i}, v_{i}\right)
$$

definida em $\Omega_{i}=\left\{\left(n_{i}, v_{i}\right) \in \mathfrak{R}^{2}: 0<n_{i} \leq 1,0<v_{i} \leq 1\right\} \cup\left\{\left(n_{i}, v_{i}\right) \in \mathfrak{R}^{2}: 0 \leq n_{i}\right.$ $\left.\leq 1, v_{i}=0\right\}, \operatorname{com} \phi_{1} \equiv \frac{\partial \phi}{\partial n_{i}}>0, \phi_{2} \equiv \frac{\partial \phi}{\partial v_{i}}>0$ e $i=1,2$. Supõe-se que esta função é tal que o subconjunto $\left\{\left(n_{i}, v_{i}\right) \in \Omega_{i}: \phi\left(n_{i}, v_{i}\right)>1 / b_{l}\right\}$ não é vazio.

Sobre estas funções barganha cabe destacar os seguintes pontos. Em primeiro lugar, foram excluídos do domínio das funções barganha os estados caracterizados por uma quantidade empregada de trabalho estritamente positiva e uma oferta nula. Nestes estados, a taxa de emprego $\left(v_{i}=l_{i} / n_{i}\right)$ é matematicamente uma indeterminação. Do ponto de vista econômico, se não existem trabalhadores na $i$-ésima ilha não faz sentido definir uma taxa 
de emprego. Já no caso em que tanto a quantidade empregada quanto a ofertada de trabalho na $i$-ésima ilha são nulas $\left(l_{i}=n_{i}=0\right)$, adotar-se-á a convenção de que $v_{i}=0$. Tal convenção implica a inclusão do ponto $(0,0)$ nos domínios das funções barganha. Em segundo lugar, admite-se que existem certas combinações de escassez de trabalho e tamanho da central sindical que implicam um poder de barganha para os trabalhadores suficiente para que estes recebam um salário maior do que sua produtividade, levando as firmas a auferirem lucros negativos. ${ }^{9}$

\section{PROPRIEDADES DINÂMICAS QUALITATIVAS BÁSICAS}

Por definição, a taxa de emprego na $i$-ésima ilha é $v_{i}=l_{i} / n_{i}$, assim:

$$
\hat{v}_{i}=\hat{l}_{i}-\hat{n}_{i}, \quad i=1,2 .
$$

Introduzindo as funções barganha (14) em (8-a) e (13), e as funções resultantes em (15), tem-se o seguinte sistema de equaçóes diferenciais que define implicitamente a função de transição de estado do modelo:

$$
\begin{aligned}
& \dot{n}_{1}=n_{1}\left(1-n_{1}\right)\left[v_{1} \phi\left(n_{1}, v_{1}\right)-v_{2} \phi\left(1-n_{1}, v_{2}\right)\right], \\
& \dot{v}_{1}=v_{1}\left\{\frac{1}{b_{k}}\left[1-\phi\left(n_{1}, v_{1}\right) b_{l}\right]-\left(1-n_{1}\right)\left[v_{1} \phi\left(n_{1}, v_{1}\right)-v_{2} \phi\left(1-n_{1}, v_{2}\right)\right]\right\}, \\
& \dot{v}_{2}=v_{2}\left\{\frac{1}{b_{k}}\left[1-\phi\left(1-n_{1}, v_{2}\right) b_{l}\right]+n_{1}\left[v_{1} \phi\left(n_{1}, v_{1}\right)-v_{2} \phi\left(1-n_{1}, v_{2}\right)\right]\right\}
\end{aligned}
$$

O espaço de estados deste sistema é definido como segue. Considerando os domínios das funções barganha, todos os estados nos quais a quantidade empregada de trabalho é estritamente positiva e a oferta de trabalho é nula

9 Nesta situação, supõe-se que o que resta no final do período de produção para as firmas é suficiente para que elas cubram parte do investimento de reposição, e que a descapitalização gerada é menor do que a depreciação do estoque de capital que ocorreria caso ele não fosse utilizado. 
em pelo menos uma das ilhas devem ser excluídos do espaço de estados do modelo. Estes estados são:

$$
\begin{aligned}
& \Theta_{1}=\left\{\left(n_{1}, v_{1}, v_{2}\right) \in \mathfrak{R}^{3}: n_{1}=0,0<v_{1} \leq 1,0 \leq v_{2} \leq 1\right\}, \\
& \Theta_{2}=\left\{\left(n_{1}, v_{1}, v_{2}\right) \in \mathfrak{R}^{3}: n_{1}=1,0 \leq v_{1} \leq 1,0<v_{2} \leq 1\right\} .
\end{aligned}
$$

Nestes estados a taxa de emprego da $i$-ésima ilha $\left(v_{i}=l_{i} / n_{i}\right)$ é, matematicamente, uma indeterminação. Do ponto de vista econômico, se não existem trabalhadores ofertando sua força de trabalho em uma ilha não pode haver trabalhadores empregados e, portanto, não faz sentido definir uma taxa de emprego nesta ilha. Portanto, o espaço de estados de (16) é definido como:

$\Theta=\left\{\left(n_{1}, v_{1}, v_{2}\right) \in \mathfrak{R}^{3}: 0 \leq n_{1} \leq 1,0 \leq v_{1} \leq 1,0 \leq v_{2} \leq 1\right\}-\left(\Theta_{1} \cup \Theta_{2}\right)$.

\subsection{Equilíbrios de Longo Prazo}

Um equilíbrio de longo prazo, isto é, um estado no qual a distribuição da população de trabalhadores não se altera e há uniformidade das taxas de lucro e dos salários entre as ilhas, pode ser classificado como: um equilíbrio de longo prazo trivial, um equilíbrio de longo prazo de estratégia pura ou um equilíbrio de longo prazo de estratégia mista. Um equilíbrio de longo prazo trivial ocorre quando as quantidades empregadas de trabalho nas ilhas são ambas nulas. Nas outras espécies de equilíbrio, as taxas de crescimento da quantidade empregada e da oferta de trabalho são iguais (mais precisamente, nulas). Num equilíbrio de longo prazo de estratégia pura todos os trabalhadores estão adotando a mesma estratégia, isto é, todos estão ofertando sua força de trabalho na ilha 1 ou na ilha 2 . Num equilíbrio de longo prazo de estratégia mista existe, simultaneamente, migração de trabalhadores para ambas as ilhas, porém numa intensidade tal que influxo e efluxo de trabalhadores em cada ilha se contrabalançam. 


\section{Equilíbrios de Longo Prazo Triviais}

Suponha que a quantidade empregada de trabalho, $l_{i}$, é nula em ambas as ilhas. Neste caso, para $n_{1}>0$ e $n_{2}>0$ tem-se $v_{1}=v_{2}=0$; se $n_{i}=0$, $i=1,2$, então, por convenção, $v_{i}=0$. Logo, todos os pontos pertencentes ao conjunto $\left.\left(n_{1}, v_{1}, v_{2}\right) \in \Theta: v_{1}=v_{2}=0\right\}$ são pontos de equilíbrio do sistema (16). Geometricamente, este continumm de equilíbrios corresponde ao intervalo real $[0,1]$ do eixo $n_{1}$ do espaço de estados.

\section{Equilíbrio de Longo Prazo de Estratégia Pura com a Ilha 1 Extinta}

Suponha que a quantidade empregada de trabalho na ilha l é nula, então $v_{1}=0$. Além disso, suponha que $v_{2}>0$, ou de modo equivalente, que $l_{2}>0$ e $n_{2}>0$. Sob tais condições, o sistema dinâmico (16) encontra-se em equilíbrio se, e somente se, as seguintes condições forem satisfeitas:

$$
n_{1}^{*}=0,1-\phi\left(1-n_{1}^{*}, v_{2}^{*}\right) b_{l}=0 .
$$

Dadas as propriedades das funções barganha, se $n_{1}^{*}=0\left(n_{2}^{*}=1\right)$ existe um único $v_{2}^{*}$ tal que $\phi\left(1, v_{2}^{*}\right)=1 / b_{l}$. Portanto, nesta situação, o sistema (16) possui um único equilíbrio de longo prazo de estratégia pura com a ilha 1 extinta, a saber: $\left(n_{1}^{*}, v_{1}^{*}, v_{2}^{*}\right)=\left(0,0, v_{2}^{*}\right)$. Intuitivamente, quando na ilha 1 a quantidade empregada de trabalho é nula e na ilha 2 a taxa de emprego é estritamente positiva, o sistema econômico como um todo estará num estado de equilíbrio de longo prazo se, e somente se, todos os trabalhadores estiverem ofertando sua força de trabalho na ilha 2 e a quantidade empregada de trabalho nesta ilha, $l_{2}$, encontrar-se num patamar suficiente para que a escassez relativa de trabalho, $v_{2}$, leve o salário ao nível que zera a taxa de lucro e, portanto, anula a taxa de crescimento da quantidade empregada de trabalho nesta ilha. 


\section{Equilíbrio de Longo Prazo de Estratégia Pura com a Ilha 2 Extinta}

Este caso é análogo ao anterior. Suponha que a quantidade empregada de trabalho na ilha 2 seja nula, isto é, que $v_{2}=0$. Se $v_{1}>0$, o sistema dinâmico (16) estará em equilíbrio se, e somente se, as seguintes condições forem observadas:

$$
1-n_{1}^{*}=0,1-\phi\left(n_{1}^{*}, v_{1}^{*}\right) b_{l}=0 .
$$

Considerando as propriedades da função barganha, se $n_{1}^{*}=1$ existe um único $v_{1}^{*}$ tal que $\phi\left(1, v_{1}^{*}\right)=1 / b_{l}$. Logo, nesta situação, o sistema (16) possui um único equilíbrio de longo prazo de estratégia pura com a ilha 2 extinta, a saber, $\left(n_{1}^{*}, v_{1}^{*}, v_{2}^{*}\right)=\left(1, v_{1}^{*}, 0\right)$.

\section{Equilíbrio de Longo Prazo de Estratégia Mista}

Suponha que as quantidades empregada e ofertada de trabalho sejam estritamente positivas em ambas as ilhas. Neste caso, o sistema dinâmico (16) estará em equilíbrio se, e somente, os salários esperados se igualarem e os níveis salariais estiverem no mesmo patamar que a produtividade do trabalho (taxas de lucro nulas), ou seja:

$v_{1}^{*} \phi\left(n_{1}^{*}, v_{1}^{*}\right)-v_{2}^{*} \phi\left(1-n_{1}^{*}, v_{2}^{*}\right)=0,1-\phi\left(n_{1}^{*}, v_{1}^{*}\right) b_{l}=0,1-\phi\left(1-n_{1}^{*}, v_{1}^{*}\right) b_{l}=0$.

Pelas duas últimas equações de (2l) infere-se que $\phi\left(n_{1}^{*}, v^{*}\right)=\phi\left(1-n_{1}^{*}, v^{*}\right)$. Logo, a primeira equação de (2l) é satisfeita se, e somente se, $v_{1}^{*}=v_{2}^{*} \equiv v^{*}$. Dessa forma, o sistema de equações que define o equilíbrio de longo prazo de estratégia mista passa a ser:

$$
\phi\left(n_{1}^{*}, v^{*}\right)=1 / b_{l}, \phi\left(1-n_{1}^{*}, v^{*}\right)=1 / b_{l} .
$$

Desde que $\phi(\cdot)$ é estritamente crescente, o sistema (22) é satisfeito se, e somente se, $n_{1}^{*}=1-n_{1}^{*}$, ou seja, se, e somente se, $n_{1}^{*}=1 / 2$. Em outras palavras, a existência e unicidade do equilíbrio de longo prazo de estratégia mista $\left(1 / 2, v^{*}, v^{*}\right)$ são garantidas pelo fato de que as curvas de nível 
implicitamente definidas por $\phi\left(n_{1}, v_{1}\right)=1 / b_{l}$ e $\phi\left(1-n_{1}, v_{1}\right)=1 / b_{l}$ se interseccionam uma única vez (cf. ponto $\mathrm{E}_{3}$ da Figura 2).

Cabe salientar que os equilíbrios de longo prazo de estratégia pura e mista apresentarão taxas de emprego estritamente menores do que um se no ponto $\left(n_{i}, 1\right)$ pertencente à curva de nível $\phi\left(n_{i}, v_{i}\right)=1 / b_{l}$ tenha-se $n_{i}<1 / 2$ para $i=1,2$. Em outros termos, as funções barganha devem apresentar a propriedade razoável de que, num mercado de trabalho em estado de pleno emprego, o tamanho da central sindical não precisa ser demasiadamente grande para que o poder de barganha dos trabalhadores, vis-à-vis ao das firmas, seja tal que o salário acordado entre as partes atinja um valor alto o suficiente para zerar a taxa de lucro. Esta condição é considerada válida daqui em diante.

FIGURA 2

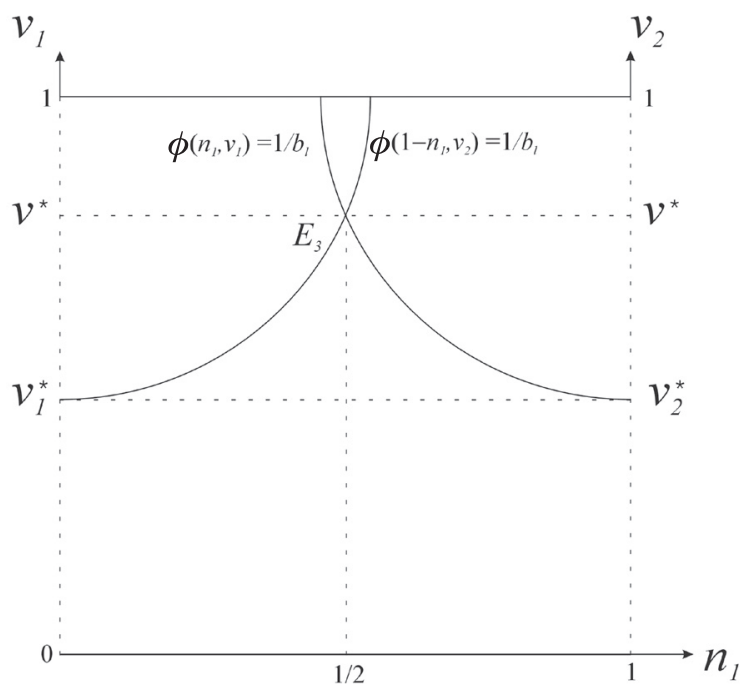

\subsection{Extinção de uma Ilha}

Quando o estado do sistema dinâmico (16) é tal que em uma das ilhas a quantidade empregada de trabalho é nula, a respectiva taxa de emprego, 
por convenção, também é nula. Dessa maneira, a outra ilha que apresenta atividade econômica propriamente dita atrairá os trabalhadores que não encontram qualquer possibilidade de emprego na ilha sem acumulação de capital.

Suponha que o estado inicial do sistema (16) é tal que a quantidade empregada de trabalho na ilha 1 seja nula. Considere a equação diferencial associada a $v_{1}$. Se $v_{1}\left(t_{0}\right)=0$, então $\dot{v}_{1}=0$ para todo $t>t_{0}$. Neste caso, o sistema (16) reduz-se a:

$$
\begin{aligned}
& \dot{n}_{1}=-n_{1}\left(1-n_{1}\right) v_{2} \phi\left(1-n_{1}, v_{2}\right), \\
& \dot{v}_{2}=v_{2}\left\{\frac{1}{b_{k}}\left[1-\phi\left(1-n_{1}, v_{2}\right) b_{l}\right]-n_{1} v_{2} \phi\left(1-n_{1}, v_{2}\right)\right\} .
\end{aligned}
$$

O espaço de estados relevante passa a ser o plano $\Theta_{3}=\left\{\left(n_{1}, v_{1}, v_{2}\right) \in \mathfrak{R}^{3}: 0\right.$ $\left.\leq n_{1}<1, v_{1}=0,0<v_{2}<1\right\} \subset \Theta$. O ponto de equilíbrio de longo prazo de estratégia pura com a ilha 1 extinta $\left(n_{1}^{*}, v_{1}^{*}, v_{2}^{*}\right)=\left(0,0, v_{2}^{*}\right)$ do sistema (16) corresponde ao ponto de equilíbrio $\left(n_{1}^{*}, v_{2}^{*}\right)=\left(0, v_{2}^{*}\right)$ do sistema (16-a).

Observe que no sistema (16-a) para todo $0<n_{1}<1$ e $v_{2}>0$ tem-se $\dot{n}_{1}<0$ $\left(\dot{n}_{2}>0\right)$, isto é, os trabalhadores ligados à ilha 1 , onde a quantidade empregada de trabalho é nula, migram progressivamente para a ilha 2. Na curva de nível definida implicitamente por $1-\phi\left(n_{2}, v_{2}\right) b_{l}=0$ (locus onde a taxa de lucro na ilha 2 é nula) e no conjunto $\left\{\left(n_{2}, v_{2}\right) \in \Omega_{2}: \phi\left(n_{2}, v_{2}\right)>1 / b_{l}\right\}$ (locus onde a taxa de lucro na ilha 2 é negativa), tem-se $\dot{v}_{2}<0$. Isto implica que a curva de demarcação $\dot{v}_{2}=0$, definida implicitamente por:

$$
\left(1 / b_{k}\right)\left[1-\phi\left(1-n_{1}, v_{2}\right) b_{l}\right]-n_{1} v_{2} \phi\left(1-n_{1}, v_{2}\right)=0
$$

está contida no conjunto $\left\{\left(n_{2}, v_{2}\right) \in \Omega_{2}: \phi\left(n_{2}, v_{2}\right)<1 / b_{l}\right\}$, cf. Figura 3 -a. 
FIGURA 3

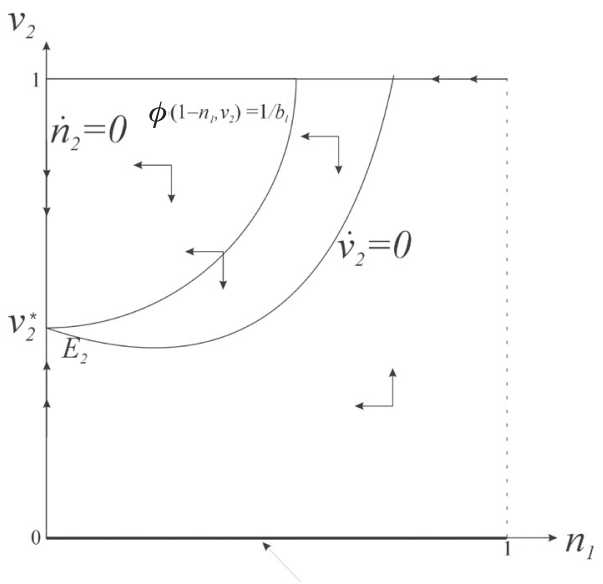

continuum de equilíbrios

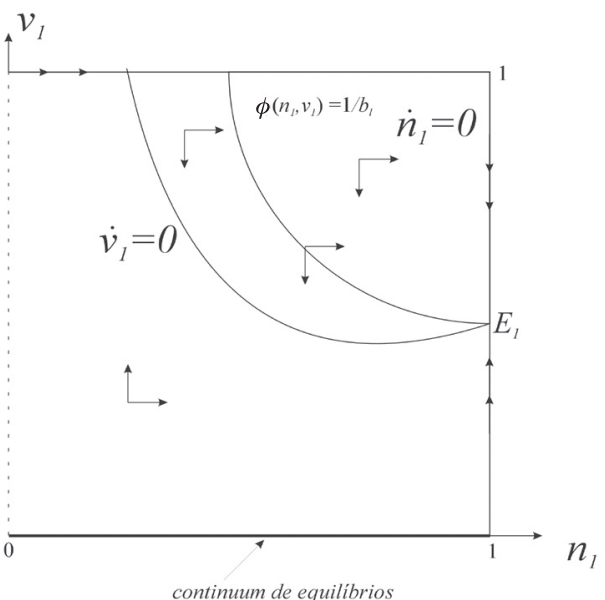

(b) Extinção da ilha 2

(a) Extinção da ilha 1

O plano de fases $\Theta_{3}$ não é um conjunto positivamente invariante. Mais precisamente, não fica garantido que o campo vetorial dado por (16-a) aponte para o interior de $\Theta_{3}$ ao longo da fronteira de pleno emprego. Entretanto, do ponto de vista econômico, as taxas setoriais de emprego não podem ser maiores que 1 . Portanto, ao atingir o pleno emprego uma ilha pode manter-se neste estado somente provisoriamente, já que a migração de trabalhadores irá aumentar o poder da central sindical e reduzir a taxa de acumulação de capital. Com base na literatura de jogos evolucionários (VEGA-REDONDO, 1996, p. 112) incorpora-se esta premissa econômica ao modelo redefinindo as equaçóes de movimento associadas às respectivas taxas de emprego, as quais passam a ser:

$$
\dot{v}_{1}=\min \left\{0, \varphi^{1}\left(n_{1}, v_{1}, v_{2}\right)\right\}, \dot{v}_{2}=\min \left\{0, \varphi^{2}\left(n_{1}, v_{1}, v_{2}\right)\right\},
$$

sendo:

$$
\begin{aligned}
& \varphi^{1}\left(n_{1}, v_{1}, v_{2}\right) \equiv v_{1}\left\{\frac{1}{b_{k}}\left[1-\phi\left(n_{1}, v_{1}\right) b_{l}\right]-\left(1-n_{1}\right)\left[v_{1} \phi\left(n_{1}, v_{1}\right)-v_{2} \phi\left(1-n_{1}, v_{2}\right)\right]\right\}, \\
& \varphi^{2}\left(n_{1}, v_{1}, v_{2}\right) \equiv v_{2}\left\{\frac{1}{b_{k}}\left[1-\phi\left(1-n_{1}, v_{2}\right) b_{l}\right]+n_{1}\left[v_{1} \phi\left(n_{1}, v_{1}\right)-v_{2} \phi\left(1-n_{1}, v_{2}\right)\right]\right\} .
\end{aligned}
$$


Portanto, no caso em análise, abaixo da fronteira de pleno emprego a dinâmica do modelo é governada por (16-a), e na fronteira de pleno emprego o sistema formado pela primeira equação diferencial em (16) e pela segunda em (24) é que passa a governar a evolução de estado do modelo. Em suma:

$$
\begin{aligned}
& \dot{n}_{1}=-n_{1}\left(1-n_{1}\right) v_{2} \phi\left(1-n_{1}, v_{2}\right), \\
& \dot{v}_{2}=\left\{\begin{array}{l}
v_{2}\left\{\frac{1}{b_{k}}\left[1-\phi\left(1-n_{1}, v_{2}\right) b_{l}\right]-n_{1} v_{2} \phi\left(1-n_{1}, v_{2}\right)\right\}, \text { se } v_{2}\left(t_{0}\right)<1, \\
\varphi^{2}\left(n_{1}, v_{1}, v_{2}\right), \text { se } v_{2}\left(t_{0}\right)=1 .
\end{array}\right.
\end{aligned}
$$

Realizando-se uma análise similar, deduz-se que a dinâmica de extinção da ilha 2 é análoga à apresentada anteriormente (cf. Figura 3-b), a saber: ${ }^{10}$

$$
\begin{aligned}
& \dot{n}_{1}=-n_{1}\left(1-n_{1}\right) v_{1} \phi\left(n_{1}, v_{1}\right), \\
& \dot{v}_{1}=\left\{\begin{array}{l}
v_{2}\left\{\frac{1}{b_{k}}\left[1-\phi\left(n_{1}, v_{1}\right) b_{l}\right]-\left(1-n_{1}\right) v_{1} \phi\left(n_{1}, v_{1}\right)\right\}, \text { se } v_{1}\left(t_{0}\right)<1, \\
\varphi^{1}\left(n_{1}, v_{1}, v_{2}\right), \text { se } v_{1}\left(t_{0}\right)=1 .
\end{array}\right.
\end{aligned}
$$

Baseado no que foi exposto até o momento, pode-se estabelecer a seguinte proposição geral com respeito à extinção de uma ilha sem atividade produtiva:

Proposição 1. (extinção de uma ilha) Suponha que a transição de estado da economia é governada pelo sistema (16-b) se $v_{1}\left(t_{0}\right)=0$ e pelo sistema (16-c) se $v_{2}\left(t_{0}\right)=0$. Se na $i$-ésima ilha há trabalhadores e a taxa de emprego é nula, então estes trabalhadores migrarão para a outra ilha até não haver qualquer trabalhador ofertando sua força de trabalho na $i$-ésima ilha. Prova: Apêndice 1.

10 Uma análise mais detalhada é encontrada em SILVEIRA (2001, p. 81). 


\subsection{A Sobrevivência de Ambas as Ilhas com Homogeneidade de Salários e de Taxas de Lucro}

No caso em que ambas as ilhas apresentam níveis de emprego estritamente positivos surge a possibilidade do processo de equilibração desencadeado pela busca de maiores salários por parte dos trabalhadores conduzir o sistema econômico a um equilíbrio de estratégia mista, no qual as ilhas coexistam e apresentem iguais salários e taxas de lucro. Na Proposição 2 estabelece-se formalmente tal condição.

Proposição 2. Suponha que a economia encontra-se numa vizinhança em torno do ponto de equilíbrio de estratégia mista $\left(1 / 2, v^{*}, v^{*}\right)$, ou seja, que ${ }^{11}$ $\left(n_{1}\left(t_{0}\right), v_{1}\left(t_{0}\right), v_{2}\left(t_{0}\right)\right) \in V\left(\left(1 / 2, v^{*}, v^{*}\right), \varepsilon\right) \subset \Theta-\left(\Theta_{3} \cup \Theta_{4}\right)$. A economia cuja transição de estado é governada pelo sistema (16) converge assintoticamente para $\left(1 / 2, v^{*}, v^{*}\right)$ se $\gamma \equiv v^{*} \frac{b_{l}}{b_{k}} \phi_{2}\left(1 / 2, v^{*}\right)+v^{*}\left(\frac{1}{b_{l}}+v^{*} \phi_{2}\left(1 / 2, v^{*}\right)\right)-v^{*} \frac{\phi_{1}\left(1 / 2, v^{*}\right)}{2}>0$.

Prova: Apêndice 2.

O impacto da migração sobre o resultado da barganha salarial ocorre por dois canais. Por um lado, uma migração líquida positiva em uma ilha tende a diminuir a taxa de emprego e, portanto, a reduzir o poder de barganha dos trabalhadores, por outro, ela aumenta a importância relativa da central sindical localizada nesta ilha no cenário macroeconômico, o que tende a dar mais poder de barganha a esta central sindical.

A condição $\gamma>0$ estabelece que, na vizinhança do equilíbrio de longo prazo de estratégia mista, o sistema econômico converge para esse equilíbrio se o efeito conjunto das forças estabilizantes, $v^{*}\left(b_{l} / b_{k}\right) \phi_{2}\left(1 / 2, v^{*}\right)+v^{*}\left(\left(1 / b_{l}\right)+v^{*} \phi_{2}\left(1 / 2, v^{*}\right)\right)$, superar o foco de instabilidade, $v^{*} \phi_{1}\left(1 / 2, v^{*}\right) / 2$. Este "efeito escala desestabilizante" representa o impacto da importância relativa das centrais sindicais no cenário macroeconômico sobre os resultados das barganhas salariais numa vizinhança do equilíbrio de longo prazo de estratégia mista. Se o tamanho

$11 V\left(\left(1 / 2, v^{*}, v^{*}\right), \varepsilon\right) \equiv\left\{\left(n_{1}, v_{1}, v_{2}\right) \in \mathfrak{R}^{3}: d\left(\left(1 / 2, v^{*}, v^{*}\right),\left(n_{1}, v_{1}, v_{2}\right)\right)<\varepsilon\right\}$, sendo $d(\cdot)$ uma distância definida numa métrica de referência e $\varepsilon$ uma constante real. 
relativo de uma central sindical afetar suficientemente o poder de barganha deste sindicato, isto poderá impedir que o sistema econômico como um todo convirja para uma situação estacionária com homogeneidade de salários e de taxas lucros. ${ }^{12}$

\section{A POSSIBILIDADE DE EMERGENNCIA DE UM CICLO COM HETEROGENEIDADE DE SALÁRIOS E DE TAXAS DE LU- CRO EM TORNO DO EQUILÍBRIO DE LONGO PRAZO DE ESTRATÉGLA MISTA}

O resultado da subseção 2.3 não exclui a possibilidade de existência de um ciclo à la Goodwin em torno do equilíbrio de longo prazo de estratégia mista. De fato, se existe um parâmetro tal que variaçóes do mesmo alteram as propriedades dinâmicas qualitativas do modelo na vizinhança do equilíbrio

12 A cadeia causal pela qual este "efeito escala desestabilizante" é transmitido pode ser detalhada como segue. No equilíbrio de longo prazo de estratégia mista, os impactos do fluxo de trabalhadores sobre os salários esperados nas ilhas 1 e 2 são, respectivamente:

$\left.\frac{\partial}{\partial n_{1}}\left(v \phi\left(n_{1}, v_{1}\right)\right)\right|_{*}=v^{*} \phi_{1}^{*}>0,\left.\frac{\partial}{\partial n_{1}}\left(v \phi\left(1-n_{1}, v_{2}\right)\right)\right|_{*}=-v^{*} \phi_{1}^{*}<0$.

Verifica-se, portanto, que os sinais destes impactos não são ambíguos. Uma expansão da população de trabalhadores na ilha 1 , ao aumentar o tamanho da respectiva central sindical, amplia o poder de barganha dos trabalhadores na ilha 1, o que resulta em ganhos reais de salário nesta ilha. É claro que esta expansão na ilha 1 contrai a população de trabalhadores na ilha 2. Isto leva a uma redução do poder de barganha dos trabalhadores na ilha 2 e, conseqüentemente, a uma redução no salário na ilha 2 .

Como o aumento da população de trabalhadores numa ilha $i$ contribui para reforçar o poder de barganha dos trabalhadores nesta ilha e enfraquecer o poder de barganha dos trabalhadores na ilha $j(j \neq i)$, o diferencial de salários esperados entre as ilhas tende a aumentar em favor da ilha $i$. Assim, na vizinhança do equilíbrio de longo prazo de estratégia mista, o impacto do fluxo de trabalhadores sobre o diferencial de salários esperados de fato possui sinal determinado, a saber:

$\left.\frac{\partial}{\partial n_{1}}\left(v \phi\left(n_{1}, v_{1}\right)\right)\right|_{*}-\left.\frac{\partial}{\partial n_{1}}\left(v \phi\left(1-n_{1}, v_{2}\right)\right)\right|_{*}=2 v^{*} \phi_{1}^{*}>0$.

Como no equilíbrio de longo prazo de estratégia mista há, em unidades de população, $n_{1}^{*}=1 / 2$ trabalhadores na ilha 1 e $n_{2}^{*}=1-n_{1}^{*}=1 / 2$ trabalhadores na ilha 2 , então o número de trabalhadores que são potenciais migrantes é $n_{2}^{*}\left(1-n_{1}^{*}\right)=1 / 4$. Logo, o impacto do fluxo de trabalhadores sobre a distribuição da população, por meio da influência da variação do tamanho das centrais sindicais sobre o poder de barganha, é dado por:

$\left.\frac{\partial \dot{n}_{1}}{\partial n_{1}}\right|_{*}=n_{1}^{*}\left(1-n_{1}^{*}\right)\left[\left.\frac{\partial}{\partial n_{1}}\left(v \phi\left(n_{1}, v_{1}\right)\right)\right|_{*}-\left.\frac{\partial}{\partial n_{1}}\left(v \phi\left(1-n_{1}, v_{2}\right)\right)\right|_{*}\right]=\frac{v \phi_{1}^{*}}{2}>0$,

que é o que se denominou anteriormente de "efeito escala desestabilizante". 
de longo prazo de estratégia mista, abre-se a possibilidade de existência de um ciclo limite, isto é, uma trajetória que forma uma órbita fechada e que é um atrator ou repulsor local ou global. Na Proposição 3, utilizando-se o conhecido teorema de bifurcação de Poincaré-Andronov-Hopf (cf. Apêndice 3), são estabelecidas condições formais que, em conjunto, garantem a existência de um ciclo limite na vizinhança do equilíbrio de estratégia mista.

Proposição 3. Suponha que a economia encontra-se numa vizinhança em torno do ponto de equilíbrio de estratégia mista $\left(1 / 2, v^{*}, v^{*}\right)$, ou seja, que $\left(n_{1}\left(t_{0}\right), v_{1}\left(t_{0}\right), v_{2}\left(t_{0}\right)\right) \in V\left(\left(1 / 2, v^{*}, v^{*}\right), \varepsilon\right) \subset \Theta-\left(\Theta_{3} \cup \Theta_{4}\right)$. A economia cuja transição de estado é governada pelo sistema (16) apresenta um ciclo limite em torno de $\left(1 / 2, v^{*}, v^{*}\right)$ se:

$$
\begin{aligned}
& \frac{\phi_{1}^{*}\left(1 / 2, v^{*}\right)}{2}-\left(\frac{1}{b_{l}}+v^{*} \phi_{2}^{*}\left(1 / 2, v^{*}\right)\right)>0 ; \\
& b_{k}>b_{k_{0}} \equiv \frac{b_{l} \phi_{2}^{*}\left(1 / 2, v^{*}\right)}{\frac{\phi_{1}^{*}\left(1 / 2, v^{*}\right)}{2}-\left(\frac{1}{b}+v^{*} \phi_{2}^{*}\left(1 / 2, v^{*}\right)\right)} .
\end{aligned}
$$

Prova: Ap ÊNDICE 4.

O SISTEM A ECONÔM ICO AP RESENTARÁ, ENTÃO, UM CICIO GOODWINIANO COM HETEROGENEIDADE DE SAIÁRIOS E DE TAXAS DE LUCRO CASO OCORRA A SEGUINTE COM B INAÇÃO DE FORÇAS P RÓXIM O DO EQUIIÍB RIO DE IONGO P RAZO DE ESTRATÉGIA M ISTA. A INFIUÊNCIA DA IM P ORTÂNCIA REIATIVA DAS CENTRAIS SINDICAIS NO CENÁRIO M ACROECONÔM ICO SOBRE OS RESUITADOS DAS B ARGANHAS SAIARIAIS DEVE SER SUHCIENTEM ENTE FORIE. ${ }^{13}$ Além disso, a economia deve apresentar uma configuração tecnológica com uma relação capital-trabalho de dimensão alta o bastante para que o impacto da barganha salarial sobre a taxa de lucro e, conseqüentemente, sobre as taxas de acumulação de capital e de crescimento do nível de emprego e do produto seja relativamente fraco. ${ }^{14}$

13 Cf. condição (i) da Proposição 3.

14 Cf. condição (ii) da Proposição 3. 
Em Silveira (2001, p. 92-97) demonstra-se a possibilidade de existência de um ciclo limite atrator em torno de $\left(1 / 2, v^{*}, v^{*}\right)$. Em outras palavras, no citado trabalho demonstrou-se que numa "situação estrutural", na qual o sistema econômico apresenta uma razão capital-produto acima da razão capital-produto crítica, $b_{k_{0}}$, a interação entre o fluxo de trabalhadores, a distribuição de renda e a acumulação de capital poderá conduzir o sistema econômico para um comportamento cíclico recorrente em torno do estado de equilíbrio de longo prazo de estratégia mista. Em outros termos, estabeleceu-se condições formais para a existência de uma bifurcação de PoincaréAndronov-Hopf supercrítica, de maneira que o ciclo que surge em torno do equilíbrio de longo prazo de estratégia mista seja localmente estável.

Na Figura 4 encontra-se um exemplo de ciclo na vizinhança do equilíbrio de longo prazo de estratégia mista. Em tal simulação, a forma funcional assumida para as funções barganha é $\phi\left(n_{i}, v_{i}\right)=\left(n_{i}^{3^{3}}+n_{i}^{2}\right) v_{i}, i=1,2$, o equilíbrio de longo prazo de estratégia mista é $\left(n_{1}^{*}, v_{1}^{*}, v_{2}^{*}\right)=(1 / 2, \boldsymbol{\theta} / \boldsymbol{\$}, \boldsymbol{\theta} / \boldsymbol{\$})$, $\rho_{1}^{*}=\rho_{2}^{*}=0$ são as taxas de lucro de equilíbrio e $\omega_{1}^{*}=\omega_{2}^{*}=5 / 19 \cong 0,26$ são os salários de equilíbrio.

\section{FIGURA 4}

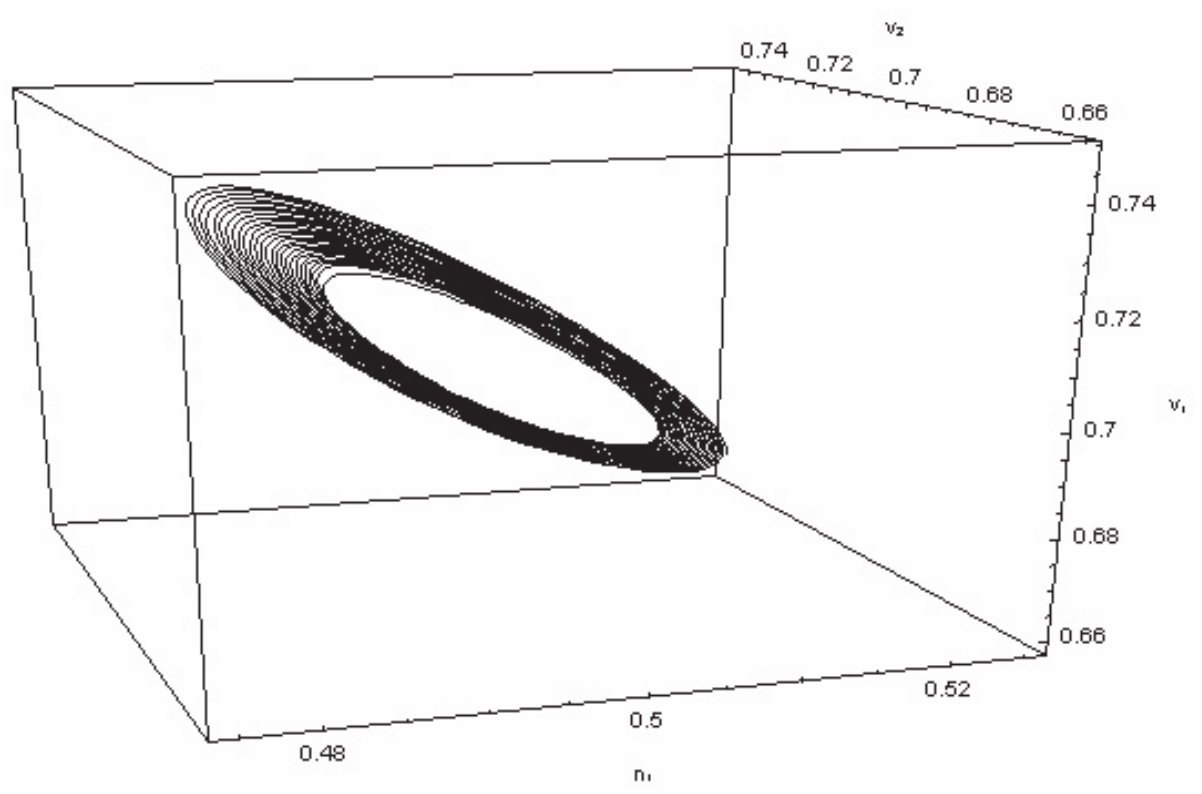

Nota: Simulação realizada com $b_{l}=3,8, b_{k}=6,5$ e $\left(n_{1}\left(t_{0}\right) v_{1}\left(t_{0}\right) v_{1}\left(t_{0}\right)=(0,5,0, \Theta, 0,1)\right.$. 
Este ciclo gerado por simulação emerge numa "situação estrutural", na qual o sistema econômico apresenta uma razão capital-produto acima da razão capital-produto crítica. Nesse tipo de configuração tecnológica, a interação entre o fluxo de trabalhadores desencadeado pela heterogeneidade de salários, a acumulação de capital e a distribuição de renda conduz o sistema econômico a um comportamento cíclico recorrente em torno do equilíbrio de longo prazo de estratégia mista.

É importante salientar que quando o sistema econômico tem seu estado inicial relativamente próximo do estado de equilíbrio de longo prazo de estratégia mista, as trajetórias das variáveis de estado do sistema (16) apresentam comportamentos cíclicos com amplitudes crescentes e que tendem assintoticamente para a amplitude de um ciclo limite na sua vizinhança. Isto significa que o "efeito escala desestabilizante" prepondera vis-à-vis as forças estabilizadoras. O oposto ocorre quando o sistema econômico tem como estado inicial um ponto relativamente mais distante do equilíbrio de longo prazo de estratégia mista.

Nessa simulação as duas ilhas estão sempre em situações econômicas opostas. Quando a ilha 1 apresenta uma tendência ascendente da taxa de emprego, a ilha 2 encontra-se numa fase de tendência descendente de sua taxa de emprego (cf. Figura 5).

FIGURA 5 - TAXAS DE EMPREGO COM $b_{l}=3,8, b_{k}=6,5$ E CONDIÇÃO INICIAL $\left(n_{1}\left(t_{0}\right) v_{1}\left(t_{0}\right) v_{1}\left(t_{0}\right)=(0,5,0, \Theta, 0,1)\right.$
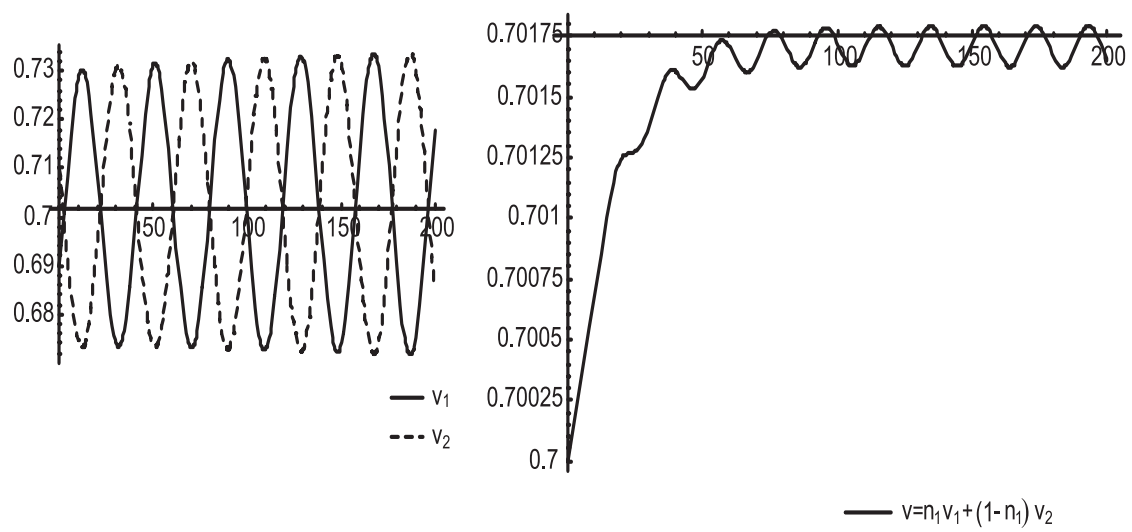
Em cada ilha há uma relação inversa entre salário e taxa de lucro, a qual decorre da hipótese sobre tecnologia, mais precisamente devido à inexistência de progresso técnico, cf. equação (13). Nas simulações há também uma relação inversa entre salário e taxa de lucro para o sistema econômico como um todo (cf. Figura 6).

FIGURA 6 - TAXA DE LUCRO MÉDIA $(\bar{\rho})$ VERSUS SALÁRIO MÉDIO $(\bar{\omega})$ COM $b_{l}=3,8, b_{k}=6,5$ E CONDIÇÃO INICIAL $\left(n_{1}\left(t_{0}\right) v_{1}\left(t_{0}\right) v_{1}\left(t_{0}\right)=(0,5,0, \theta, 0,1)\right.$

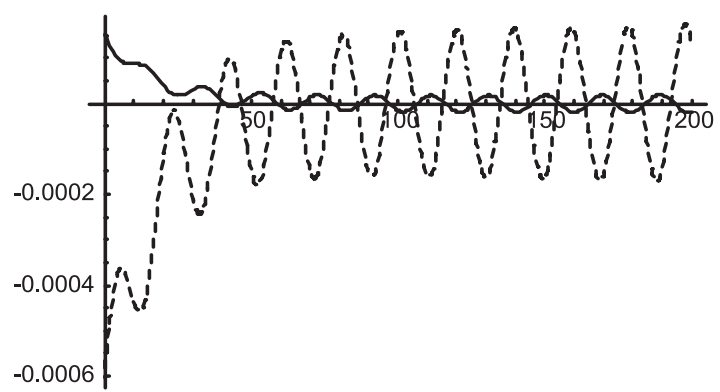

Finalmente, observa-se que as taxas de emprego, os salários e taxas de lucro nas ilhas apresentam flutuações com maiores amplitudes que as respectivas flutuações médias (cf. Figuras 7 e 8). Em outras palavras, as simulações apresentam ciclos macroeconômicos mais suaves que os ciclos microeconômicos.

FIGURA 7 - SALÁRIOS COM $b_{l}=3,8, b_{k}=6,5$ E CONDIÇÃO INICIAL

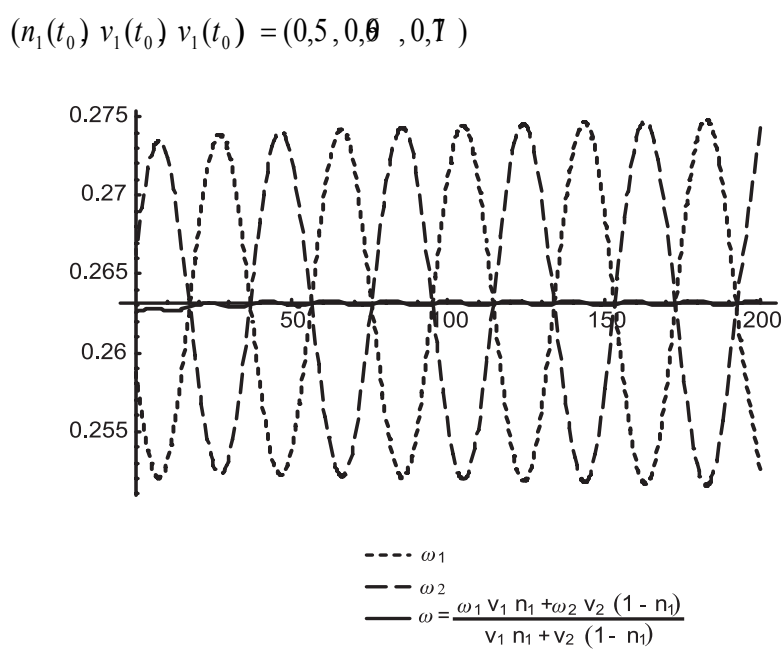


FIGURA 8 - TAXAS DE LUCRO COM $b_{l}=3,8, b_{k}=6,5$ E CONDIÇÃO

INICIAL $\left(n_{1}\left(t_{0}\right) v_{1}\left(t_{0}\right) v_{1}\left(t_{0}\right)=(0,5,0, \Theta, 0,1)\right.$

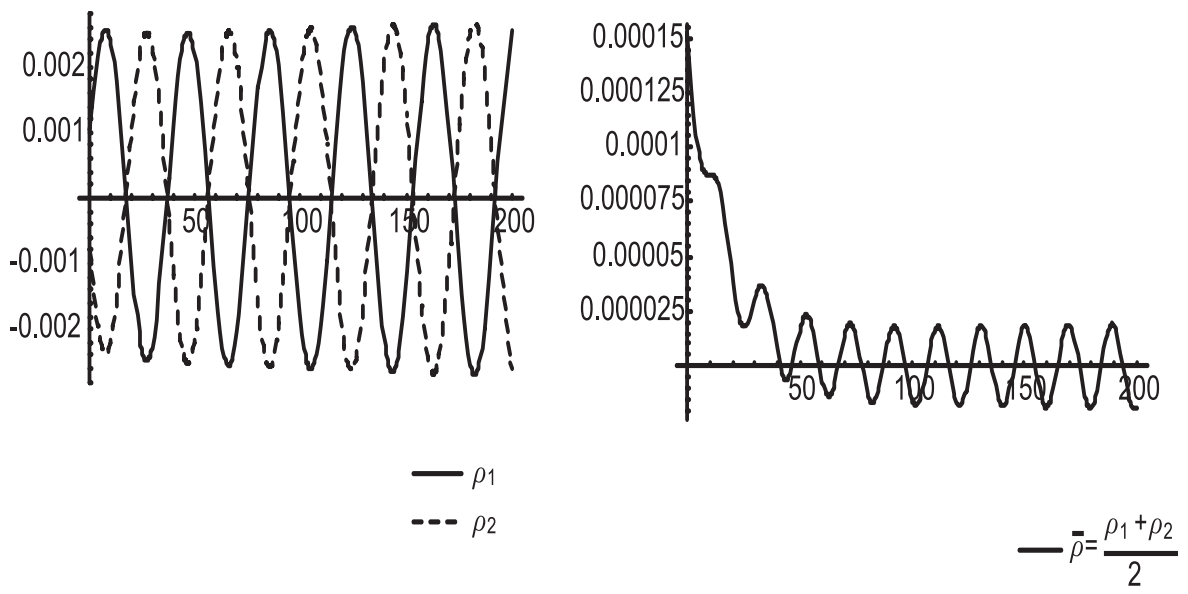

\section{CONSIDERAÇÕES FINAIS}

Neste trabalho apresentou-se um modelo econômico com características clássicas/goodwinianas composto por dois mercados de trabalho distintos, cujos diferenciais de salários desecadeiam um fluxo migratório de trabalhadores num ambiente de racionalidade limitada. Neste cenário, avaliou-se a possibilidade de existência de ciclos com heterogeneidade de salários e de taxas de lucro. Provou-se que quando o sistema econômico apresenta uma relação capital-trabalho acima de um valor crítico, existe a possibilidade de emergência de ciclos goodwinianos com heterogeneidade de salários e de taxas de lucro. Em outras palavras, sob a hipótese plausível de que a relação capital-trabalho tende a aumentar historicamente (um dos fatos estilizados kaldorianos), o modelo sugere que a partir de um determinado estágio do progresso tecnológico as economias capitalistas podem apresentar flutuaçóes cíclicas de cunho clássico, como no modelo de Goodwin (1967).

Cabe salientar que existe a possibilidade do modelo econômico aqui desenvolvido apresentar, de maneira alternada, fases de estabilidade assintótica (convergência para um estado de equilíbrio de longo prazo) e fases cíclicas (flutuações em torno de um estado de equilíbrio de longo prazo). 
Essa seqüência de fases não apresenta uma periodicidade, pois a duração de cada uma delas dependerá de choques tecnológicos que alteram a situação estrutural do sistema econômico. Os choques tecnológicos podem ser interpretados como eventos históricos que podem deslocar o sistema econômico de uma situação estrutural assintoticamente estável para uma situação estrutural cíclica (e vice-versa). Cabe ressaltar que estas mudanças de técnicas produtivas não são responsáveis per se pela manutenção das flutuações. Os ciclos são gerados pelo próprio modus operandi do sistema econômico. Evidentemente, os resultados obtidos dependem da específica formalização do processo migratório de trabalhadores que foi adotada e, em particular, do efeito escala na função barganha.

\section{APÊNDICE 1 - PROVA DA PROPOSIÇÃO I}

Suponha que $\left(n_{1}\left(t_{0}\right), v_{1}\left(t_{0}\right), v_{2}\left(t_{0}\right)\right) \in \Theta_{3}=\left\{\left(n_{1}, v_{1}, v_{2}\right) \in \mathfrak{R}^{3}: 0 \leq n_{1}<1, v_{1}=0,0\right.$ $\left.<v_{2}<1\right\} \subset \Theta$. O estado $\left(n_{1}^{*}, v_{2}^{*}\right)=\left(0, v_{2}^{*}\right)$ é o único ponto de equilíbrio do sistema (16-b) em $\Theta_{3}$. A matriz Jacobiana da linearização em torno de $\left(n_{1}^{*}, v_{2}^{*}\right)=\left(0, v_{2}^{*}\right)$ é dada por:

$$
J\left(0, v_{2}^{*}\right)=\left[\begin{array}{c:c}
-v_{2}^{*} \frac{1}{b_{l}} & 0 \\
\hdashline v_{2}^{*}\left(\frac{b_{l}}{b_{k}} \phi_{1}^{*}-\frac{1}{b_{l}} v_{2}^{*}\right) & -v_{2}^{*} \frac{b_{l}}{b_{k}} \phi_{2}^{*}
\end{array}\right],
$$

na qual $\phi_{1}^{*} \equiv \phi_{1}\left(1, v_{2}^{*}\right)$ e $\phi_{2}^{*} \equiv \phi_{2}\left(1, v_{2}^{*}\right)$. A equação característica associada a esta matriz é $\lambda^{2}+a_{1} \lambda+a_{0}=0$, na qual:

$a_{0}=\operatorname{det} J\left(0, v_{2}^{*}\right)=\frac{\left(v_{2}^{*}\right)^{2} \phi_{2}^{*}}{b_{k}}>0$ e $a_{1}=-\operatorname{tr} J\left(0, v_{2}^{*}\right)=v_{2}^{*}\left(\frac{1}{b_{l}}+\frac{b_{l}}{b_{k}} \phi_{2}^{*}\right)>0$.

De (A2) infere-se que o ponto de equilíbrio $\left(n_{1}^{*}, v_{2}^{*}\right)=\left(0, v_{2}^{*}\right)$ é um sorvedouro da linearização em torno deste, já que os autovalores têm parte real negativa. Dessa forma, pelo teorema de Hartman-Grobman conclui-se que o sistema (16-b) é localmente assintoticamente estável. Na fronteira de $\Theta_{3}$ o campo vetorial definido por (16-b) aponta para o interior de $\Theta_{3}$. Isto 
significa que se o sistema econômico estiver na vizinhança do equilíbrio de longo prazo $\left(n_{1}^{*}, v_{1}^{*}, v_{2}^{*}\right)=\left(0,0, v_{2}^{*}\right)$ e pertencente a $\Theta_{3}$, este convergirá e a ilha 1 será extinta.

Suponha que $\left(n_{1}\left(t_{0}\right), v_{1}\left(t_{0}\right), v_{2}\left(t_{0}\right)\right) \in \Theta_{4}=\left\{\left(n_{1}, v_{1}, v_{2}\right) \in \mathfrak{R}^{3}: 0<n_{1} \leq 1,0\right.$. $\left.<v_{1}<1, v_{2}=0\right\} \subset \Theta$. O estado $\left(n_{1}^{*}, v_{1}^{*}\right)=\left(1, v_{1}^{*}\right)$ é o único ponto de equilíbrio do sistema (16-c) em $\Theta_{4}$. A matriz Jacobiana da linearização em torno de $\left(n_{1}^{*}, v_{1}^{*}\right)$ é dada por:

$$
J\left(0, v_{2}^{*}\right)=\left[\begin{array}{c:c}
-v_{1}^{*} \frac{1}{b_{l}} & 0 \\
\hdashline v_{1}^{*}\left(-\frac{b_{l}}{b_{k}} \phi_{1}^{*}+\frac{1}{b_{l}} v_{1}^{*}\right)_{1} & -v_{1}^{*} \frac{b_{l}}{b_{k}} \phi_{2}^{*}
\end{array}\right],
$$

na qual $\phi_{1}^{*} \equiv \phi_{1}\left(1, v_{1}^{*}\right)$ e $\phi_{2}^{*} \equiv \phi_{2}\left(1, v_{1}^{*}\right)$. A equação característica associada a esta matriz é $\lambda^{2}+a_{1} \lambda+a_{0}=0$, na qual:

$a_{0}=\operatorname{det} J\left(1, v_{1}^{*}\right)=\frac{\left(v_{1}^{*}\right)^{2} \phi_{2}^{*}}{b_{K}}>0$ e $a_{1}=-\operatorname{tr} J\left(1, v_{1}^{*}\right)=\left(\frac{1}{b_{l}}+\frac{b_{l}}{b_{k}} \phi_{2}^{*}\right)>0$.

De (A4) infere-se que o ponto de equilíbrio $\left(n_{1}^{*}, v_{1}^{*}\right)=\left(1, v_{1}^{*}\right)$ é um sorvedouro da linearização em sua vizinhança, pois os autovalores associados possuem partes reais negativas. Assim, com base no teorema de HartmanGrobman, infere-se que o sistema (16-c) é localmente assintoticamente estável. Na fronteira de $\Theta_{3}$ o campo vetorial definido por (16-c) aponta para o interior de $\Theta_{4}$. Isto significa que se o sistema econômico estiver na vizinhança do equilíbrio de longo prazo $\left(n_{1}^{*}, v_{1}^{*}, v_{2}^{*}\right)=\left(1, v_{1}^{*}, 0\right)$ e pertencente a $\Theta_{4}$, este convergirá e a ilha 2 será extinta.

\section{APÊNDICE 2 - PROVA DA PROPOSIÇÃO 2}

A matriz Jacobiana da linearização em torno do equilíbrio de longo prazo de estratégia mista $\left(1 / 2, v^{*}, v^{*}\right)$ do sistema (16) é dada por: 


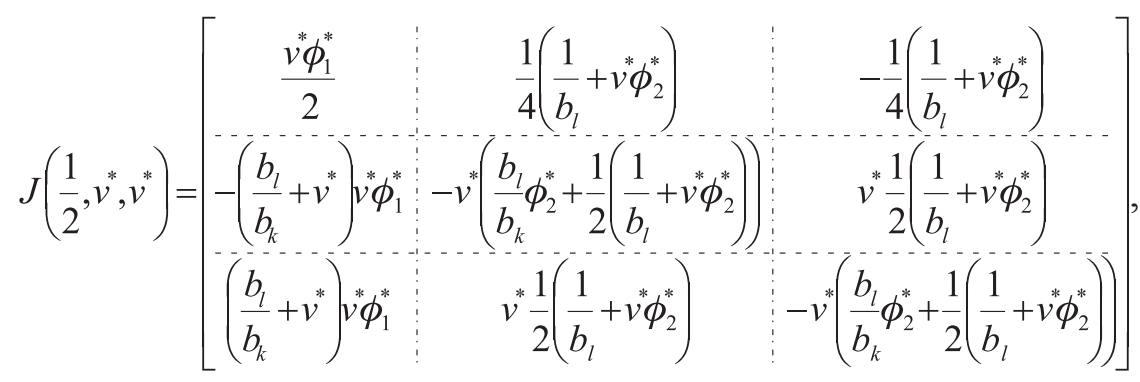

na qual $\phi_{1}^{*} \equiv \phi_{1}\left(1 / 2, v^{*}\right)$ e $\phi_{2}^{*} \equiv \phi_{2}\left(1 / 2, v^{*}\right)$. A equação característica associada a esta matriz é $\lambda^{3}+a_{2} \lambda^{2}+a_{1} \lambda+a_{0}=0$, cujos autovalores (raízes características) são:

$$
\lambda_{1}=-\beta<0, \lambda_{2,3}=-\frac{\gamma}{2} \pm \sqrt{-\alpha+\left(-\frac{\gamma}{2}\right)^{2}},
$$

sendo $\alpha \equiv\left(v^{*} \frac{\phi_{1}^{*}}{2}\right) \frac{1}{b_{k}}, \beta \equiv\left(v \frac{b_{l}}{b_{k}} \phi_{2}^{*}\right)$ e $\gamma \equiv v^{*} \frac{b_{l}}{b_{k}} \phi_{2}^{*}+v^{*}\left(\frac{1}{b_{l}}+v^{*} \phi_{2}^{*}\right)-v^{*} \frac{\phi_{1}^{*}}{2}$. Decorre diretamente das premissas sobre a tecnologia e a função barganha que $\beta>0$. Assim, os autovalores (A6) apresentam parte real estritamente negativa se, e somente se, $\gamma>0$.

\section{APÊNDICE 3 - TEOREMA DE BIFURCAÇÃO DE POINCARÉ- ANDRONOV-HOPF}

O teorema adiante está enunciado, sob a denominação de "teorema de bifurcação de Hopf”, em Guckenheimer e Holmes (1990, p. 151):

Theorem 3.4.2 (Hopf [1942]). Suppose that the system $\dot{x}=f_{\mu}(x), x \in \mathfrak{R}^{n}$, $\mu \in \mathfrak{R}$ has an equilibrium $\left(x_{0}, \mu_{0}\right)$ at which the following properties are satisfied:

(HI) $D_{x} f_{\mu_{0}}\left(x_{0}\right)$ has a simple pair of pure imaginary eigenvalues and no other eigenvalues with zero real parts. 
Then (HI) implies that there is an smooth curve of equilibria $(x(\mu), \mu)$ with $x$ $\left(\mu_{0}\right)-x_{0}$. The eigenvalues $\lambda(\mu), \bar{\lambda}(\mu)$ of $D_{x} f_{\mu_{0}}(x(\mu))$ which are imaginary at $\mu=\mu_{0}$ vary smoothly with $\mu$. If, moreover,

(H2) $\left.\frac{d}{d \mu}(\operatorname{Re} \lambda(\mu))\right|_{\mu=\mu_{0}}=d \neq 0$,

then there is a unique three-dimensional center manifold passing through $\left(x_{0}, \mu_{0}\right)$ in $\mathfrak{R}^{n} \times \mathfrak{R}$ and a smooth system of coordinates (preserving the planes $\mu=$ const.) for which the Taylor expansion of degree 3 on the center manifold is given by

$$
\begin{aligned}
& \dot{x}=\left(d \mu+a\left(x^{2}+y^{2}\right)\right) x-\left(\omega+c \mu+b\left(x^{2}+y^{2}\right)\right) y, \\
& \dot{y}=\left(\omega+c \mu+b\left(x^{2}+y^{2}\right)\right) x+\left(d \mu+a\left(x^{2}+y^{2}\right)\right) y,
\end{aligned}
$$

which is expressed in polar coordinates as

$$
\begin{aligned}
& \dot{r}=\left(d \mu+a r^{2}\right) r, \\
& \dot{\theta}=\left(\omega+c \mu+b r^{2}\right) .
\end{aligned}
$$

If $a \neq 0$, there is a surface of periodic solutions in the center manifold which has quadratic tangency with the eigenspace of $\lambda\left(\mu_{0}\right), \bar{\lambda}\left(\mu_{0}\right)$ agreeing to second order with the paraboloid $\mu=-(a / d)\left(x^{2}+y^{2}\right)$. If $a<0$ then these periodic solutions are stable limit cycles [bifurcação de Poincaré-Andronov-Hopf supercrítica], while if $a>0$, the periodic solutions are repelling [bifurcação de Poincaré-Andronov-Hopf subcrítica].

\section{APÊNDICE 4 - PROVA DA PROPOSIÇÃO 3}

Pelo teorema de Hartman-Grobman e considerando (A6) o equilíbrio ( $1 / 2$, $\left.v^{*}, v^{*}\right)$ é localmente instável se a seguinte condição for satisfeita:

$$
\gamma \equiv v^{*} \frac{b_{l}}{b_{k}} \phi_{2}^{*}+v^{*}\left(\frac{1}{b_{l}}+v^{*} \phi_{2}^{*}\right)-\frac{v^{*} \phi_{1}^{*}}{2}<0,
$$


na qual $\phi_{1}^{*} \equiv \phi_{1}\left(1 / 2, v^{*}\right)$ e $\phi_{2}^{*} \equiv \phi_{2}\left(1 / 2, v^{*}\right)$. Como $v^{*} \frac{b_{l}}{b_{k}} \phi_{2}^{*}>0$, uma condição necessária, embora não suficiente, para a instabilidade local do equilíbrio de longo prazo de estratégia mista é:

$$
\frac{v^{*} \phi_{1}^{*}}{2}-v^{*}\left(\frac{1}{b_{l}}+v^{*} \phi_{2}^{*}\right)>0
$$

Supondo-se que (A8) é satisfeita pelo sistema (16), o impacto de variações da taxa de emprego na $i$-ésima ilha sobre a respectiva taxa de lucro,

$$
\left.\frac{\partial}{\partial v_{i}}\left(\frac{1}{b_{k}}\left(1-\phi\left(n_{i}, v_{i}\right) b_{l}\right)\right)\right|_{*}=-\frac{b_{l}}{b_{k}} \phi_{2}^{*}<0, \quad i=1,2
$$

passa a determinar a estabilidade do sistema econômico na vizinhança do equilíbrio $\left(1 / 2, v^{*}, v^{*}\right)$. O impacto das variações das taxas de emprego setoriais sobre suas respectivas taxas de lucro, dada a forma funcional das funções barganha, depende da tecnologia a que está sujeito o sistema econômico, isto é, depende da razão capital-trabalho $b_{k} / b_{l}$. Para uma dada produtividade do trabalho $1 / b_{l}$, o valor da razão capital-produto que estabelece a fronteira entre estabilidade e instabilidade local do equilíbrio $\left(1 / 2, v^{*}, v^{*}\right)$ é dado por:

$$
b_{k_{0}}=b_{l} \phi_{2}^{*} / \frac{\phi_{1}^{*}}{2}-\left(\frac{1}{b_{l}}+v^{*} \phi_{2}^{*}\right) .
$$

Quando o parâmetro de bifurcação $b_{k}$ é igual ao valor de bifurcação $b_{k_{0}}$ a parte real dos autovalores complexos conjugados da matriz Jacobiana (A5) anula-se, isto é, $\operatorname{Re}\left(\lambda_{2,3}\left(b_{k_{0}}\right)\right)=-\gamma / 2=0$, pois $\gamma=0$. Logo, $\left(n_{1}^{*}, v_{1}^{*}, v_{1}^{*} ; b_{k}\right)=\left(1 / 2, v^{*}, v^{*} ; b_{k 0}\right)$ é um ponto de bifurcação do sistema (16).

Portanto, se (A8) é satisfeita, o sistema (16), no ponto de bifurcação (1/2, $\left.v^{*}, v^{*}\right)$, satisfaz a condição $(\mathrm{Hl})$ do teorema de bifurcação de Poincaré-Andronov-Hopf (cf. Apêndice 3), isto é, apresenta um autovalor real negativo e um par de autovalores imaginários puros conjugados, dados por: 
$\lambda_{1}\left(b_{k_{0}}\right)=-\left[\frac{v^{*} \phi_{1}^{*}}{2}-v^{*}\left(\frac{1}{b_{l}}+v^{*} \phi_{2}^{*}\right)\right]<0, \quad \lambda_{2,3}\left(b_{k_{0}}\right)= \pm i \sqrt{\frac{\phi_{1}^{*}}{2 b_{l} \phi_{2}^{*}}\left[\frac{v^{*} \phi_{1}^{*}}{2}-v^{*}\left(\frac{1}{b_{l}}+v^{*} \phi_{2}^{*}\right)\right]}$.

Considerando (A6), o efeito de uma variação da razão capital-produto sobre a parte real dos autovalores complexos conjugados da matriz Jacobiana (A5) é dado por:

$$
\frac{d \operatorname{Re}\left(\lambda_{2,3}\left(b_{k}\right)\right)}{d b_{k}}=\frac{d}{d b_{k}}\left(-\frac{1}{2} \gamma\right)=\frac{v^{*} b_{l} \phi_{2}^{*}}{2 b_{k}^{2}} .
$$

Avaliando esta derivada no valor de bifurcação, isto é, substituindo (A10) em (Al2), infere-se, utilizando (A8), que esta não é nula, ou seja:

$$
\left.\frac{d \operatorname{Re}\left(\lambda_{2,3}\left(b_{k}\right)\right)}{d b_{k}}\right|_{b_{k}=b_{k_{0}}}=\frac{v^{*}}{2 b_{l} \phi_{2}^{*}}\left(\frac{1}{b_{l}}+v \phi_{2}^{*}-\frac{\phi_{1}^{*}}{2}\right)^{2}>0 .
$$

Assim, fica satisfeita a condição (H2) do teorema de bifurcação de Poincaré-Andronov-Hopf (cf. Apêndice 3), isto é, (Al3) garante que há uma mudança na estrutura qualitativa da solução do sistema (16) quando este cruza o ponto de bifurcação.

\section{REFERÊNCIAS BIBLIOGRÁFICAS}

Goodwin, R. M. A growth cycle. In: FEINSTEIN, C. H. (ed.), Socialism, capitalism and economic growth: Essays presented to Maurice Dobb. Cambridge: Cambridge University Press, 1967.

Goodwin, R. M.; Krüger, M.; Vercelli, A. (eds.). Nonlinear models of fluctuating growth. Berlin: Springer-Verlag, 1984. (Lecture notes in economics and mathematical systems, n. 228).

Guckenheimer, John; Holmes, Philip. Nonlinear oscillations, dynamical systems, and bifurcations of vector fields. 3. ed. New York: Springer-Verlag, 1990.

Hofbauer, J.; Sigmund, K. Evolutionary games and population dynamics. Cambridge: Cambridge University Press, 1998.

SATO, Yoshikazu. Marx-Goodwin Growth cycles in a two-sector economy. Zeitschrift für Nationalökonomie, v. 45, n. 1, p. 21-39, 1985.

SAMUELSON, L. Evolution games and equilibrium selection. Cambridge: MIT Press, 1997. 
SILVEIRA, Jaylson Jair da. Ciclos goodwinianos e o processo de concorrência num ambiente de racionalidade limitada: uma análise a partir da teoria dos jogos evolucionários. 2001. Tese (Doutorado) - Instituto de Pesquisas Econômicas, Universidade de São Paulo, São Paulo.

VEGA-REDONDO, F. Evolution, games and economic behaviour. Oxford: Oxford University Press, 1996.

Weibull, J. W. Evolutionary game theory. Cambridge: MIT Press, 1995.

Este artigo é uma versão concisa de um dos modelos desenvolvidos em minha tese (SILVEIRA, cap. 2 e 3, 2001). Agradeço os comentários feitos pelos professores Décio K. Kadota, Eleutério F. S. Prado, Jean-Luc S. Rosinger, João R. Sanson e Jorge E. C. Soromenho na ocasião da defesa da tese e ao professor Ronald Hillbrecht também a Aquino L. Espíndola por sua ajuda no desenho das figuras não geradas por simulação numérica. Naturalmente, os erros remanescentes são de minha exclusiva responsabilidade.

(Recebido em maio de 2002. Aceito para publicação em julho de 2003). 